05,04

\title{
Особенности катионного распределения и магнитных свойств гексаферритов $\mathrm{BaFe}_{12-x} \mathrm{Y}_{x} \mathrm{O}_{19}$
}

\author{
(C) В.Г. Костишин ${ }^{1}$, В.В. Коровушкин ${ }^{1}$, И.М. Исаев ${ }^{1}$, А.Ю. Миронович ${ }^{1}$, С.В. Труханов ${ }^{2}$, \\ B.А. Турченко ${ }^{3,4,5}$, К.А. Астапович ${ }^{2}$, А.В. Труханов ${ }^{1,29}$
}

${ }^{1}$ Национальный исследовательский технологический университет (МИСиС),

Москва, Россия

${ }^{2}$ НПЦ НАН Беларуси по материаловедению,

Минск, Беларусь

${ }^{3}$ Лаборатория нейтронной фризики им. И.М. Франка, Объединенный институт ядерных исследований,

Дубна, Россия

${ }^{4}$ Южно-Уральский государственный университет,

Челябинск, Россия

${ }^{5}$ Донецкий ффизико-технический институт им. А.А. Галкина НАНУ,

Киев, Украина

^E-mail: truhanov86@mail.ru

Поступила в Редакцию 6 сентября 2020 г.

В окончательной редакции 6 сентября 2020 г.

Принята к публикации 9 сентября 2020 г.

\begin{abstract}
Проведены исследования гексаферрита $\mathrm{BaY}_{x} \mathrm{Fe}_{12-x} \mathrm{O}_{19}(0.1 \leq x \leq 1.2)$ с помощью мёссбауэровской спектроскопии, магнитометрии и рентгенографии. Показана невысокая изоморфная емкость гексаферрита, что приводит при $x=0.6$ к фазовому расслоению с образованием $\mathrm{BaFe}_{2} \mathrm{O}_{4}$, и $\mathrm{Y}_{3} \mathrm{Fe}_{5} \mathrm{O}_{12}$. Данные мёссбауэровской спектроскопии показали, что ионы $\mathrm{Y}^{3+}$ в изученном диапазоне замещений входят в позицию $12 k$ с образованием неэквивалентного положения $12 k^{\prime}$ за счет обрыва двух магнитных связей $\mathrm{Fe}(12 k)-\mathrm{O}-\mathrm{Fe}(12 k)$ в триаде октаэдров $12 k$ с их заменой на $\mathrm{Fe}(12 k)-\mathrm{O}-\mathrm{Y}(12 k)$. Показано, что гексаферрит $\mathrm{BaY}_{x} \mathrm{Fe}_{12-x} \mathrm{O}_{19}$ является менее магнитотвердым, нежели $\mathrm{BaFe}_{12-x} \mathrm{Al}_{x} \mathrm{O}_{19}$.
\end{abstract}

Ключевые слова: гексаферрит бария, иттрий, мёссбауэровская спектроскопия, магнитометрия, рентгенография, удельная намагниченность, коэрцитивная сила, коэффициент замещения, неэквивалентные положения, структурные позиции.

DOI: 10.21883/FTT.2021.02.50468.187

\section{1. Введение}

Гексагональные ферриты типа $M$, в частности, $\mathrm{BaFe}_{12} \mathrm{O}_{19}$, находят все большее применение в различных областях электротехнической и электронной промышленности. Они используются как постоянные малогабаритные магниты в устройствах магнитоэлектроники и микросистемной техники, СВЧ-устройствах, как запоминающие элементы, радиопоглощающие материалы идр. Это объясняется их широкой изоморфной емкостью и разнообразием изоморфных элементов, которые позволяют в широком диапазоне варьировать магнитные, электрические, механические, химические и другие свойства ферритов. Гексаферриты $M$-типа имеют кристаллическую структуру, изоморфную природному минералу магнетоплюмбиту $\mathrm{PbFe}_{12} \mathrm{O}_{19}$. Ионы железа в структуре гексаферрита заселяют пять кристаллографических позиций: $2 a, 2 b, 4 f_{1}, 4 f_{2}$ и $12 k$. Позиции $2 a, 4 f_{2}$ и $12 k$ являются октаэдрическими, $4 f_{1}$ - тетраэдрической, а $2 b$ образует бипирамиду. Полиэдры $4 f_{1}$ и $2 a$ расположены в шпинельном блоке $(S), 4 f_{2}$ и $2 b-$ в гексагональном $(R)$, а $12 k$ - на границе шпинельного и гексагонального блоков $(R S)[1]$. В незамещенном гек- саферрите $\mathrm{BaFe}_{12} \mathrm{O}_{19}$ имеет место коллинеарная магнитная структура, в которой магнитные моменты позиций $12 k, 2 a$ и $2 b$ направлены в одну сторону, а $4 f_{1}$ и $4 f_{2}$ в другую, антипараллельно [2], что приводит к нескомпенсированному антиферромагнетизму (ферримагнетизму). Ослабление этих взаимодействий за счет замещения ионов $\mathrm{Fe}^{3+}$ обеих групп немагнитными и/или слабомагнитными ионами металлов приводит к уменьшению результирующего магнитного момента. В литературе имеется большой набор данных по изовалентным одноэлементным изоморфным замещениям в гексаферритах, при которых сохраняется баланс электронейтральности зарядов [3-7].

Рассматривая вхождение иттрия в решетку гексаферрита, многие авторы для более выраженного изменения магнитных и электрических свойств исследуют парное вхождение наряду с иттрием других элементов, как трехвалентных, так и двухвалентных. Так в работе [8], изучая гексаферрит $\mathrm{Sr}_{1-x} \mathrm{Mn}_{x} \mathrm{Fe}_{12-x} \mathrm{Y}_{x} \mathrm{O}_{19}$ с помощью мёссбауэровской спектроскопии, авторы отмечают увеличение изомерного сдвига ионов в позициях $4 f_{1}$ и $2 a$ при увеличении $x$ от 0.1 до 0.5. При этом квадрупольное расщепление ионов в позиции $4 f_{2}$ при $x=0.3$ меняет знак, 
площади секстетов $12 k$ и $2 a$ уменьшаются, а площадь $4 f_{2}$ возрастает. В спектре появляется дополнительный секстет, площадь которого возрастает с увеличением степени замещения $x$. Авторы считают, что ионы $\mathrm{Mn}^{2+}$ не только замещают ионы $\mathrm{Ba}^{2+}$, но при $x \geq 0.3$ входят в позицию $12 k$ и являются возможной причиной изменения знака квадрупольного расщепления $\mathrm{Fe}^{3+}$ позиции $4 f_{2}$, смежной с $12 k$. Однако как реализуется при этом зарядовый баланс, не совсем понятно.

В работе [9] авторы исследовали механизм электропроводности переменного тока и диэлектрические свойства гексаферритов $\mathrm{BaBi}_{x} \mathrm{La}_{x} \mathrm{Y}_{x} \mathrm{Fe}_{12-3 x} \mathrm{O}_{19}$ $(0 \leq x \leq 0.33)$ в диапазоне частот от $1 \mathrm{~Hz}$ до $1 \mathrm{MHz}$ в интервале температур $20-120^{\circ} \mathrm{C}$ с использованием метода комплексной импедансной спектроскопии. Целью работы было исследование влияния электрических свойств гексаферритов на процессы микроволнового поглощения, однако влияние магнитных свойств при этом не рассматривалось.

Магнитные свойства гексаферрита того же состава были изучены в работе [10], в которой была установлена высокая удельная $\left(53.7-67.4 \mathrm{~A} \cdot \mathrm{m}^{2} / \mathrm{kg}\right)$ и остаточная намагниченности. Коэрцитивная сила с повышением степени замещения в гексаферрите уменьшается до $2.18 \mathrm{~A} / \mathrm{m}$, что позволяет считать гексаферрит достаточно магнитотвердым материалом.

Авторы [11] для изучения магнитных свойств гексаферрита $\mathrm{SrBi}_{x} \mathrm{La}_{x} \mathrm{Y}_{x} \mathrm{Fe}_{12-3 x} \mathrm{O}_{19}(0 \leq x \leq 0.33)$ использовали рентгенографию, магнитометрию и мёссбауэровскую спектроскопию. Установили однофазный состав гексаферрита, ферримагнитную природу наночастиц и уменьшение коэрцитивной силы с увеличением содержания легирующих ионов. По данным мёссбауэровской спектроскопии определены изменения ширины линий, изомерного сдвига, квадрупольного расщепления, относительной площади и магнитного поля на ядрах $\mathrm{Fe}^{57}$ при замещениях $\mathrm{Bi}^{3+}, \mathrm{La}^{3+}$ и $\mathrm{Y}^{3+}$.

Непосредственное вхождение в структуру гексаферрита ионов $\mathrm{Y}^{3+}$ было исследовано в работах авторов $[12,13]$. Используя рентгенографию и магнитные измерения при исследовании гексаферрита $\mathrm{BaY}_{x} \mathrm{Fe}_{12-x} \mathrm{O}_{19}$ $(0 \leq x \leq 1.0)$, авторы [12] отметили рост параметров элементарной ячейки $a, b$ и $c$ с увеличением $x$ от 0 до 0.6 в связи с большим радиусом $\mathrm{Y}^{3+}$ нежели у $\mathrm{Fe}^{3+}$, но после 0.6 все параметры уменьшаются. Намагниченность насыщения увеличивается до $x=0.4$, а затем уменьшается, что авторы объясняют замещениями в позициях $4 f_{1}$ и $4 f_{2}$. Однако трудно представить, что крупный ион $\mathrm{Y}^{3+}$ входит в тетраэдр. При этом коэрцитивная сила резко возрастает при $x$ от 0 до 0.4, а затем увеличивается незначительно. Полученные результаты, безусловно, представляют интерес, но многие моменты не находят убедительного объяснения, например, возрастание коэрцитивной силы вместе с намагниченностью в интервале $x$ до 0.4 и уменьшение параметров элементарной ячейки после 0.6. Авторы [13] изучали гексаферрит $\mathrm{Ba}_{(1-x)} \mathrm{Y}_{x} \mathrm{Fe}_{12} \mathrm{O}_{19}$ с более низкими концентрациями иттрия $(x=0,0.02,0.05,0.08,0.1,0.13)$. При этом они отмечают увеличение отношения параметров элементарной ячейки $c / a$ гексаферрита, связывая эти изменения с разными ионными радиусами $\mathrm{Y}^{3+}$ и $\mathrm{Fe}^{3+}$, но предполагают замену ионов $\mathrm{Ba}^{2+}$ ионами $\mathrm{Y}^{3+}$. Намагниченность сначала увеличивается в диапазоне $x$ от 0 до 0.04, а затем уменьшается. Коэрцитивная сила сначала резко увеличивается с $2.1 \mathrm{kOe}$ до $3.5 \mathrm{kOe}$ при $x=0.04$, а затем монотонно растет до $3.7 \mathrm{kOe}$ при $x=0.13$. При этом во всех образцах, начиная с $x=0.05$, методом рентгенографии фиксируется примесь гематита.

Проведенный краткий обзор по гексаферритам типа $M$, легированных только иттрием, показывает, что, с одной стороны, слишком мало подобных работ, а с другой стороны, анализ катионного распределения ионов железа и иттрия в структуре $M$-типа не является полным и имеются разные интерпретации результатов.

Целью настоящей работы является изучение катионного распределения в гексаферритах $\mathrm{BaFe}_{12-x} \mathrm{Y}_{x} \mathrm{O}_{19}$, сравнение пределов изоморфизма иттрия с другими элементами и оценка его влияния на магнитные свойства с использованием мёссбауровской спектроскопии, магнитометрии и рентгенографии.

\section{2. Объекты и методы исследования}

Объектами исследования служили образцы поликристаллических гексаферритов бария $\mathrm{BaFe}_{12-x} \mathrm{Y}_{x} \mathrm{O}_{19}$, где $x=0.1,0.3,0.6,0.9$ и 1.2. Образцы изготовлялись по керамической технологии из оксидов $\mathrm{Fe}_{2} \mathrm{O}_{3}, \mathrm{Y}_{2} \mathrm{O}_{3}$ и карбоната $\mathrm{BaCO}_{3}$ квалификации „ОСЧ“. Предварительный исходный состав подвергался синтезирующему обжигу на воздухе при $1200^{\circ} \mathrm{C}(6 \mathrm{~h})$, а затем спекался при $1300^{\circ} \mathrm{C}(6 \mathrm{~h})$. После спекания образцы медленно охлаждались в печи $\left(\sim 100^{\circ} / \mathrm{h}\right)$ [14]. Мессбауэровские спектры образцов $\mathrm{BaFe}_{12-x} \mathrm{Y}_{x} \mathrm{O}_{19}$ регистрировались на спектрометре Ms-1104 Em с постоянным ускорением, с числом точек 512, с источником $\gamma$-излучения $\mathrm{Co}^{57}$ в матрице хрома, при комнатной температуре. Изомерный (химический) сдвиг рассчитывался относительно $\alpha$-Fe. Использовались порошковые пробы крупностью $0.05-0.07 \mathrm{~mm}$ из спеченных ферритов. Математическая обработка спектров проводилась по программе Univem Ms. Магнитные параметры: удельную намагниченность $\sigma_{s}$, остаточную намагниченность $\sigma_{r}$, коэрцитивную силу $H_{c}$ и форму петли гистерезиса измеряли на вибрационном магнитометре VSM-250 в магнитном поле напряженностью $20 \mathrm{kOе}$ при $300 \mathrm{~K}$. Фазовый состав образцов определялся с помощью дифрактометра Rigaku Ultima IV с фокусировкой по Брэггу-Брентано на $\mathrm{Cu} K_{\alpha}$ излучении с использованием графитового монохроматора на дифрагированном пучке. Использовался режим сканирования по точкам, диапазон углов 20: 20-140, шаг 0.05 . 

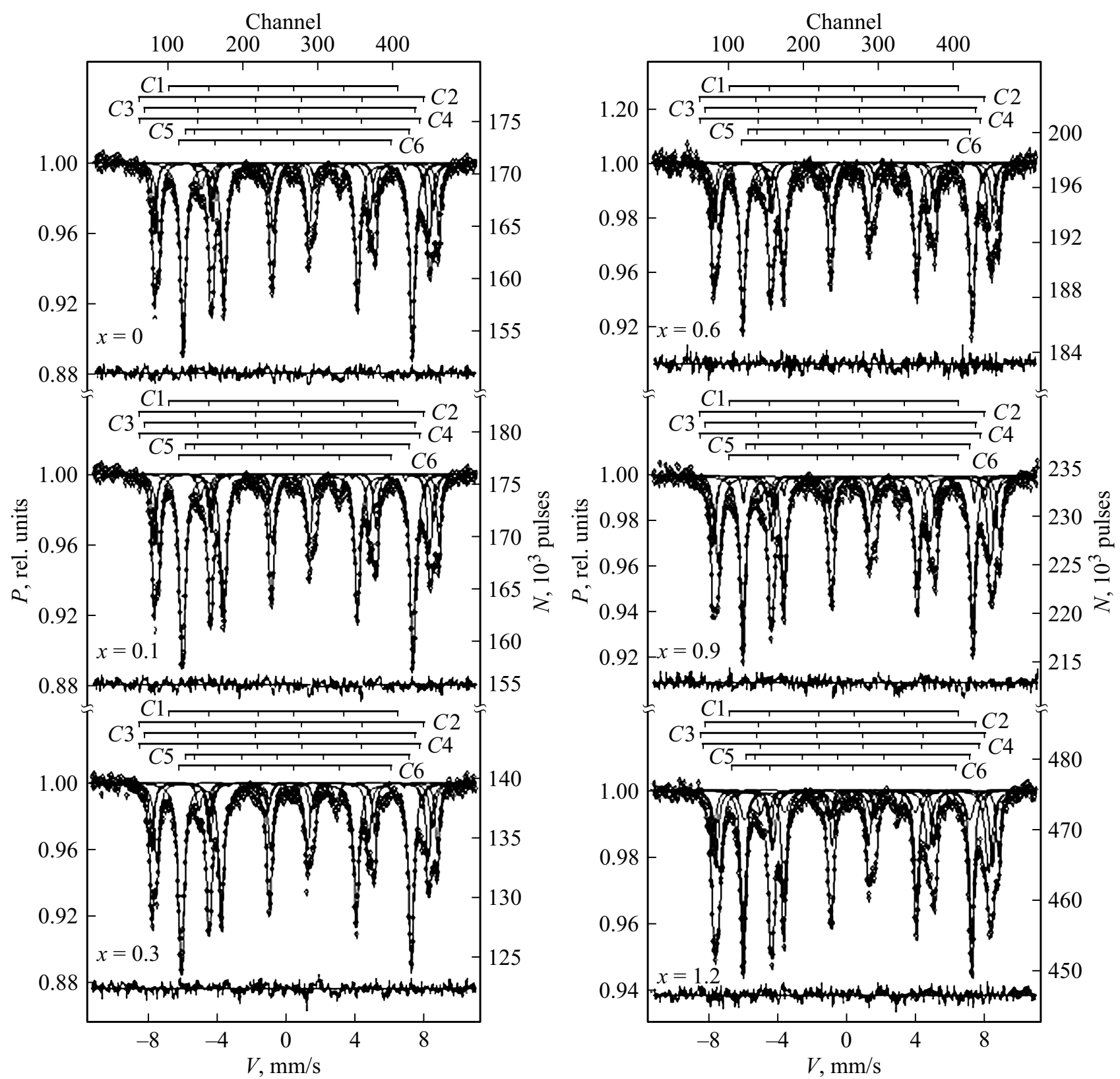

Рис. 1. Мессбауэровские спектры гексагональных ферритов $\mathrm{BaFe}_{12-x} \mathrm{Y}_{x} \mathrm{O}_{19}(x=0,0.1,0.3,0.6,0.9,1.2)$.

\section{3. Результаты и обсуждение}

Мессбауэровские спектры образцов гексаферритов $\mathrm{BaFe}_{12-x} \mathrm{Y}_{x} \mathrm{O}_{19}$ приведены на рис. 1. По программе Univem Ms все спектры были разложены на составляющие секстеты. Модель разложения задавалась из следующих соображений. В замещенных ферритах выделялись секстеты, принадлежащие ионам железа основных пяти позиций, с параметрами незамещенного гексаферрита и визуально определялись положения дополнительных секстетов. Наилучший вариант обработки по программе определялся по параметру $\min \chi^{2}$ при сохранении физического смысла мёссбауэровских параметров. По уменьшению интегральных интенсивностей пиков сек- стетов устанавливались позиции в структуре, в которые входят ионы иттрия, и какие магнитные связи косвенного магнитного обмена $\mathrm{Fe}-\mathrm{O}-\mathrm{Fe}$ обрываются. При этом учитывалось, что в случае неэквивалентных положений ионов Fе при вхождении замещающего иона в установленную позицию могут образовываться магнитные связи с соседними полиэдрами. Принималось, что вхождение иона иттрия в позицию $12 k$ может образовывать два неэквивалентных положения $12 k^{\prime}$ за счет сочленения трех октаэдров $12 k$. В соответствии с приведенными соображениями мёссбауэровские спектры всех образцов были разложены на шесть секстетов, что означает появление одного неэквивалентного положения иона $\mathrm{Fe}^{3+}$. Такое разложение обеспечило наилучшие значения 
Таблица 1. Параметры мёссбауэровских спектров $\mathrm{BaFe}_{12-x} \mathrm{Y}_{x} \mathrm{O}_{19}$

\begin{tabular}{|c|c|c|c|c|c|c|}
\hline $\begin{array}{c}\text { Образец } \\
\mathrm{BaFe}_{12-x} \mathrm{Y}_{x} \mathrm{O}_{19} \\
\end{array}$ & $\begin{array}{c}\text { Компонента } \\
\text { спектра }\end{array}$ & $\begin{array}{c}\text { Изомерный } \\
\text { сдвиг } \delta, \mathrm{mm} / \mathrm{s}\end{array}$ & $\begin{array}{c}\text { Квадрупольное } \\
\text { расщепление } \Delta, \mathrm{mm} / \mathrm{s}\end{array}$ & $\begin{array}{c}\text { Магнитные } \\
\text { поля } H_{e f f}, \mathrm{kOe} \\
\end{array}$ & $\begin{array}{c}\text { Площади } \\
\text { компонент } S, \%\end{array}$ & $\begin{array}{c}\text { Ширина линии } \\
\Gamma, \mathrm{mm} / \mathrm{s} \\
\end{array}$ \\
\hline$x=0$ & $\begin{array}{l}C 1(12 k) \\
C 2\left(4 f_{1}\right) \\
C 3\left(4 f_{2}\right) \\
C 4(2 a) \\
C 5(2 b)\end{array}$ & $\begin{array}{l}0.35 \\
0.26 \\
0.38 \\
0.34 \\
0.28\end{array}$ & $\begin{array}{l}0.42 \\
0.22 \\
0.20 \\
0.01 \\
2.21\end{array}$ & $\begin{array}{l}414 \\
489 \\
516 \\
507 \\
400\end{array}$ & $\begin{array}{r}50.5 \\
19.8 \\
16.8 \\
7.5 \\
5.3\end{array}$ & $\begin{array}{l}0.32 \\
0.31 \\
0.29 \\
0.26 \\
0.30\end{array}$ \\
\hline$x=0.1$ & $\begin{array}{l}C 1(12 k) \\
C 2\left(4 f_{2}\right) \\
C 3\left(4 f_{1}\right) \\
C 4(2 a) \\
C 5(2 b) \\
C 6\left(12 k^{\prime}\right)\end{array}$ & $\begin{array}{l}0.36 \\
0.39 \\
0.29 \\
0.35 \\
0.30 \\
0.43\end{array}$ & $\begin{array}{l}0.42 \\
0.20 \\
0.20 \\
0.00 \\
2.23 \\
0.02\end{array}$ & $\begin{array}{l}413 \\
514 \\
489 \\
506 \\
401 \\
379\end{array}$ & $\begin{array}{c}48.1 \\
13.4 \\
19.6 \\
12.3 \\
5.05 \\
1.1\end{array}$ & $\begin{array}{l}0.37 \\
0.27 \\
0.35 \\
0.37 \\
0.3 \\
0.21\end{array}$ \\
\hline$x=0.3$ & $\begin{array}{l}C 1(12 k) \\
C 2\left(4 f_{2}\right) \\
C 3\left(4 f_{1}\right) \\
C 4(2 a) \\
C 5(2 b) \\
C 6\left(12 k^{\prime}\right) \\
\end{array}$ & $\begin{array}{l}0.36 \\
0.39 \\
0.27 \\
0.33 \\
0.29 \\
0.44 \\
\end{array}$ & $\begin{array}{l}0.42 \\
0.20 \\
0.20 \\
0.01 \\
2.21 \\
0.45 \\
\end{array}$ & $\begin{array}{l}413 \\
515 \\
489 \\
506 \\
401 \\
380 \\
\end{array}$ & $\begin{array}{r}47.3 \\
13.5 \\
19.7 \\
12.9 \\
5.7 \\
0.8 \\
\end{array}$ & $\begin{array}{l}0.37 \\
0.27 \\
0.35 \\
0.41 \\
0.31 \\
0.23\end{array}$ \\
\hline$x=0.6$ & $\begin{array}{l}C 1(12 k) \\
C 2\left(4 f_{2}\right) \\
C 3\left(4 f_{1}\right) \\
C 4(2 a) \\
C 5(2 b) \\
C 6\left(12 k^{\prime}\right)\end{array}$ & $\begin{array}{l}0.36 \\
0.38 \\
0.27 \\
0.35 \\
0.30 \\
0.43\end{array}$ & $\begin{array}{l}0.42 \\
0.19 \\
0.21 \\
0.04 \\
2.15 \\
0.27\end{array}$ & $\begin{array}{l}414 \\
516 \\
489 \\
503 \\
398 \\
383\end{array}$ & $\begin{array}{r}42.0 \\
11.9 \\
21.0 \\
16.0 \\
7.0 \\
2.1\end{array}$ & $\begin{array}{l}0.35 \\
0.24 \\
0.41 \\
0.42 \\
0.45 \\
0.23\end{array}$ \\
\hline$x=0.9$ & $\begin{array}{l}C 1(12 k) \\
C 2\left(4 f_{2}\right) \\
C 3\left(4 f_{1}\right) \\
C 4(2 a) \\
C 5(2 b) \\
C 6\left(12 k^{\prime}\right)\end{array}$ & $\begin{array}{l}0.35 \\
0.39 \\
0.26 \\
0.36 \\
0.32 \\
0.36 \\
\end{array}$ & $\begin{array}{l}0.41 \\
0.17 \\
0.22 \\
0.01 \\
2.23 \\
0.35 \\
\end{array}$ & $\begin{array}{l}413 \\
516 \\
488 \\
501 \\
400 \\
382 \\
\end{array}$ & $\begin{array}{r}41.6 \\
13.7 \\
22.9 \\
14.8 \\
4.9 \\
2.1 \\
\end{array}$ & $\begin{array}{l}0.36 \\
0.30 \\
0.42 \\
0.31 \\
0.29 \\
0.23 \\
\end{array}$ \\
\hline$x=1.2$ & $\begin{array}{l}C 1(12 k) \\
C 2\left(4 f_{2}\right) \\
C 3\left(4 f_{1}\right) \\
C 4(2 a) \\
C 5(2 b) \\
C 6\left(12 k^{\prime}\right)\end{array}$ & $\begin{array}{l}0.35 \\
0.39 \\
0.25 \\
0.35 \\
0.29 \\
0.35\end{array}$ & $\begin{array}{l}0.42 \\
0.17 \\
0.21 \\
0.04 \\
2.16 \\
0.40\end{array}$ & $\begin{array}{l}412 \\
514 \\
485 \\
499 \\
400 \\
405\end{array}$ & $\begin{array}{r}34.5 \\
11.9 \\
23.1 \\
18.5 \\
4.9 \\
7.1\end{array}$ & $\begin{array}{l}0.36 \\
0.28 \\
0.46 \\
0.36 \\
0.34 \\
0.38\end{array}$ \\
\hline
\end{tabular}

критерия Пирсона $\chi^{2}$. При этом возрастание степени замещения $x$ сопровождалось увеличением интегральной интенсивности дополнительного секстета. Полученные параметры секстетов изомерный (химический) сдвиг $\delta(\mathrm{mm} / \mathrm{s})$, квадрупольное расщепление $\Delta(\mathrm{mm} / \mathrm{s})$, магнитное поле на ядрах $\mathrm{Fe}^{57}\left(H_{e f f}, \mathrm{kOe}\right)$, площади компонент спектра $S$ (rel.\%), ширина резонансной линии $\Gamma(\mathrm{mm} / \mathrm{s})$ и их соответствие занимаемым позициям, приведены в табл. 1. Для сопоставления в таблице приведены параметры одного из незамещенных гексаферритов бария.

Оценивая мёссбауэровские параметры (табл. 1) как для незамещенных, так и для замещенных гексаферритов, можно отметить стабильность изомерного сдвига $\delta$ $(0.35-0.36 \mathrm{~mm} / \mathrm{s})$ от ионов $\mathrm{Fe}^{3+}$ в позиции $12 k$ и квадрупольное расщепление $(0.41-0.42 \mathrm{~mm} / \mathrm{s})$ для секстетов 1 . Для ионов $\mathrm{Fe}^{3+}$ в октаэдрической позиции $4 f_{2}$ средняя величина $\delta$ составила $0.39 \mathrm{~mm} / \mathrm{s}$, а для $\mathrm{Fe}^{3+}$ в позиции $12 k$ и $2 a-0.35 \mathrm{~mm} / \mathrm{s}$. Поскольку величина изомерного сдвига возрастает с увеличением ионности связи, то для ионов $\mathrm{Fe}^{3+}$ в позиции $4 f_{2}$ характерна большая его величина, нежели для ионов $\mathrm{Fe}^{3+}$ в позициях $12 k$ и $2 a$.

Существенно меньшие значения величины $\delta$ показывают ионы $\mathrm{Fe}^{3+}$ в тетраэдрической позиции $4 f_{1}(0.25-0.29 \mathrm{~mm} / \mathrm{s})$ и бипирамидальной позиции $2 b$ $(0.29 \mathrm{~mm} / \mathrm{s})$ в соответствии с [15], что объясняется большей ковалентностью связи между ионами железа за счет меньшего объема полиэдров $4 f_{1}$ и $2 b$. Квадрупольное расщепление $\Delta$ ионов $\mathrm{Fe}^{3+}$ зависит только от градиента электрического поля лигандов и увеличивается при отклонении симметрии полиэдров от идеальной [16]. Поэтому значительные искажения симметрии полиэдра $2 b$ объясняют квадрупольное расщепление уровней яд- 


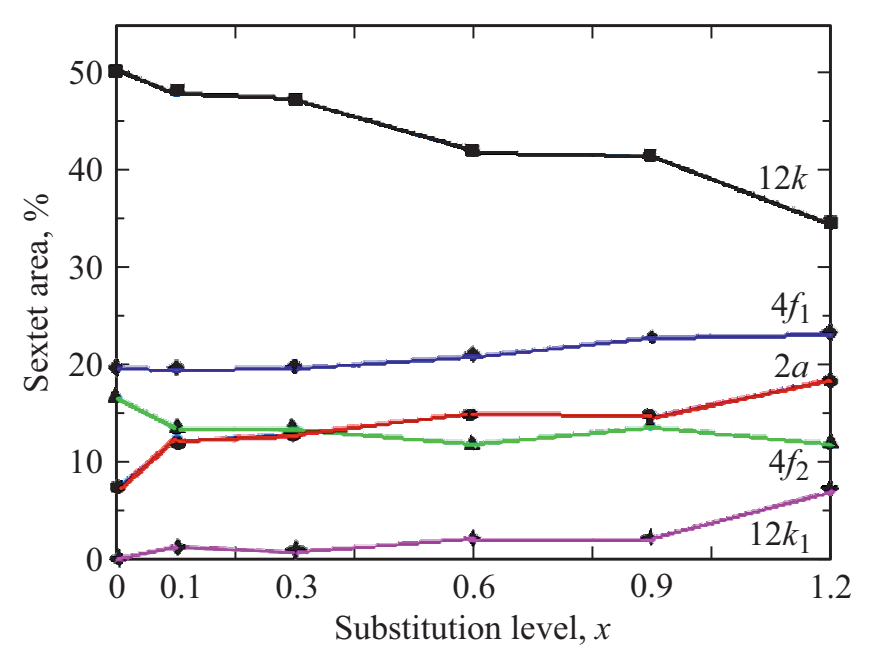

Рис. 2. Зависимость интегральных интенсивностей секстетов мёссбауэровских спектров $\mathrm{BaFe}_{12-x} \mathrm{Y}_{x} \mathrm{O}_{19}$ от степени замещения $x$.

pa $\mathrm{Fe}^{57}(2.15-2.27 \mathrm{~mm} / \mathrm{s})$, в то время как для самого симметричного октаэдра $2 a \Delta$ изменяется в пределах $(0-0.04 \mathrm{~mm} / \mathrm{s})$. Магнитные поля на ядрах $\mathrm{Fe}^{57}$ всех основных позиций изменяются незначительно. Некоторое уменьшение с ростом $x$ наблюдается для магнитного поля на ядрах $\mathrm{Fe}^{57}$ в позиции $2 a$, а при $x=1.2$ - скачок до $410 \mathrm{kOе}$ для $12 k^{\prime}$. Это указывает на то, что замещения в гексаферрите носят ограниченный характер. Об этом можно судить и при визуальной оценке формы спектров, которая не регистрирует существенных изменений.

Изменения в спектрах образцов можно увидеть лишь при сопоставлении площадей секстетов. Как видно из табл. 1, существенное изменение претерпевает интегральная интенсивность от ионов $\mathrm{Fe}^{3+}$ в позициях $12 k, 2 a, 4 f_{1}$ и дополнительного секстета $12 k^{\prime}$. Некоторое уменьшение площадей секстетов, соответствующих ионам $\mathrm{Fe}^{3+}$ бипирамиды, по сравнению с теоретическими значениями во всех образцах объясняется различием амплитуд колебаний ионов $\mathrm{Fe}^{3+}$ в этом полиэдре, но не замещениями. Из сопоставления площадей секстетов всех пяти основных позиций видно, что наибольшее изменение претерпела площадь секстета, соответствующего ионам в позиции $12 k$. Это послужило основанием считать, что ионы иттрия в основном входят в эту позицию, а дополнительный секстет, обозначенный как $12 k^{\prime}$, образуется за счет обрыва двух магнитных связей $\mathrm{Fe}(12 k)-\mathrm{O}-\mathrm{Fe}(12 k)$ в триаде октаэдров $12 k$ с их заменой на $\mathrm{Fe}(12 k)-\mathrm{O}-\mathrm{Y}(12 k)$. Динамику изменения интегральных интенсивностей секстетов, соответствующих ионам $\mathrm{Fe}^{3+}$, можно проследить на рис. 2.

Из зависимостей интегральной интенсивности секстетов мёссбауэровских спектров видно, что в них можно выделить два диапазона. Один включает значения $x$ в интервале $0.1-0.9$, а второй - в интервале 0.9-1.2. В первом интервале значительные изменения заключаются в уменьшении интенсивностей секстетов $12 k$ и незначительном увеличении интенсивностей секстетов $4 f_{1}, 2 a$ и $12 k^{\prime}$. Во втором интервале можно наблюдать более существенные изменения интенсивностей секстетов: более резкое уменьшение интенсивности секстета $12 k$, небольшое уменьшение $4 f_{2}$ и увеличение $12 k^{\prime}$, $4 f_{1}$ и $2 a$. Это можно объяснить, по нашим соображениям, следующим образом. Замещения в позиции $12 k$ обусловили появление секстета $12 k^{\prime}$ и привели к обрыву магнитной связи $\mathrm{Fe}(12 k)-\mathrm{O}-\mathrm{Fe}\left(4 f_{2}\right)$, в результате чего интенсивность секстета, соответствующего ионам $\mathrm{Fe}^{3+}$ в позиции $4 f_{2}$, уменьшилась. Некоторое уменьшение магнитного поля на ядрах $\mathrm{Fe}^{57}$ в позиции $2 a$ указывает на то, что частично обрываются связи $\mathrm{Fe}(12 k)-\mathrm{O}-\mathrm{Fe}(2 a)$, а наличие примесных фаз объясняет увеличение интенсивностей $4 f_{1}$ и $2 a$. Сопоставление интенсивностей гексаферрита типа $M$ и феррита-граната показало, что положения пиков 1 и 6 от октаэдрических ионов $\mathrm{Fe}^{3+}$ феррита-граната очень близки с положениями пиков, соответствующих ионам $\mathrm{Fe}^{3+}$ в позиции $4 f_{1}$ гексаферрита, и именно это приводит к увеличению площади $4 f_{1}$, в отличие от данных работы [13], где авторы считают, что иттрий входит в тетраэдрическую позицию. Но понижения заселенности позиции $4 f_{1}$ в наших экспериментах не наблюдается ни при одном составе гексаферрита, кроме того, мы считаем, что ион $\mathrm{Y}^{3+}$ с большим радиусом $(1.06 \AA)$ не может входить в тетраэдр с меньшим объемом, чем октаэдр. Аналогично, возможно наложение на площадь $2 a$ пиков от ферриташпинели $\mathrm{BaFe}_{2} \mathrm{O}_{4}$, что приводит к ее увеличению в образцах с $x=0.3-1.2$. Влияние железосодержащих примесей на интегральные интенсивности некоторых секстетов подтвердили рентгенографические измерения.

Полученные рентгенограммы образцов $\mathrm{BaFe}_{12-x} \mathrm{Y}_{x} \mathrm{O}_{19}$ приведены на рис. 3, а в табл. 2 -

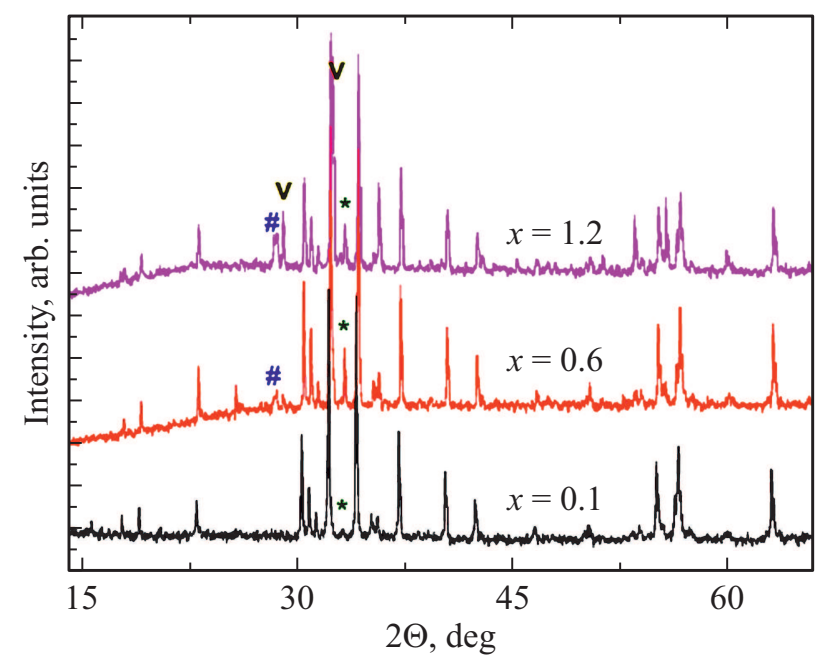

Рис. 3. Общий вид рентгеновских дифракционных спектров образцов $\mathrm{BaFe}_{12-x} \mathrm{Y}_{x} \mathrm{O}_{19}(x=0.1,0.6$ и 1.2$)$, полученных при комнатной температуре. Отмечены самые интенсивные линии, принадлежащие примесным фазам: $*-\mathrm{Fe}_{2} \mathrm{O}_{3}$, \#- $\mathrm{BaFe}_{2} \mathrm{O}_{4}$, $\mathrm{v}-\mathrm{Y}_{3} \mathrm{Fe}_{5} \mathrm{O}_{12}$. 
Таблица 2. Количественный фазовый состав образцов твердого раствора $\mathrm{BaFe}_{12-x} \mathrm{Y}_{x} \mathrm{O}_{19}(x=0.1-1.2)$ в зависимости от концентрации ионов $\mathrm{Y}$

\begin{tabular}{c|c|c|c|c}
\hline $\begin{array}{c}\text { Конц. } \\
\mathrm{Y}^{3+}, x\end{array}$ & $\begin{array}{c}\text { Фаза } \\
\mathrm{BaFe}_{12-x} \mathrm{Y}_{x} \mathrm{O}_{19}, \%\end{array}$ & $\begin{array}{c}\text { Фаза } \\
\mathrm{Fe}_{2} \mathrm{O}_{3}, \%\end{array}$ & $\begin{array}{c}\text { Фаза } \\
\mathrm{BaFe}_{2} \mathrm{O}_{4}, \%\end{array}$ & $\begin{array}{c}\text { Фаза } \\
\mathrm{Y}_{3} \mathrm{Fe}_{5} \mathrm{O}_{12}, \%\end{array}$ \\
\hline 0.1 & 96.7 & 3.3 & 0 & 0 \\
0.3 & 95.7 & 4.3 & 0 & 0 \\
0.6 & 88.6 & 4.5 & 6.9 & 0 \\
0.9 & 75.5 & 3.9 & 6.3 & 14.3 \\
1.2 & 68.1 & 3.3 & 6.5 & 22.1
\end{tabular}

зарегистрированные примесные фазы и количественные их содержания. Небольшая фаза оксида $\mathrm{Fe}_{2} \mathrm{O}_{3}$ (3.3-4.5\%) присутствует в образцах всех составов и существенного изменения в мёссбауэровские спектры не вносит, при этом иттрий полностью входит в решетку гексаферрита, но при $x=0.6$ появляется фаза $\mathrm{BaFe}_{2} \mathrm{O}_{4}$, содержание которой мало изменяется по мере увеличения степени замещения, но в ней может содержаться примесь иттрия. Более существенные изменения состава образцов появляются при $x=0.9$ когда рентгенография определяет появление фазы феррита-граната $\mathrm{Y}_{3} \mathrm{Fe}_{5} \mathrm{O}_{12}(14.3 \%)$, показывающее, что не весь иттрий стал входить в решетку гексаферрита и наступил предел его изоморфного вхождения. При $x=1.2$ содержание феррита-граната еще более возрастает и достигает 22\%. Согласно полученным результатам, можно заключить, что изоморфные замещения в гексаферрите $\mathrm{BaFe}_{12-x} \mathrm{Y}_{x} \mathrm{O}_{19}$ носят ограниченный характер и изоморфная его емкость существенно ниже, чем при замещениях $\mathrm{Al}$, In, $\mathrm{Ga}, \mathrm{Ti}, \mathrm{Zn}$ и другими элементами. Причиной этого является, по-видимому, большой ионный радиус иона $\mathrm{Y}^{3+}(1.06 \AA)$, в отличие от перечисленных выше элементов, а также различие в их электронной конфигурации. Присутствие примесной фазы иттриевого феррита-граната подтверждает увеличение интегральной интенсивности секстета $4 f_{1}$, интенсивность которого также возрастает с увеличением $x$. При этом следует отметить, что, судя по мёссбауэровским спектрам, возрастание интегральной интенсивности секстета $4 f_{1}$ начинается уже при $x=0.6$, и предел вхождения ионов $\mathrm{Y}^{3+}$, возможно, ниже, чем $x=0.9$. Слабый рефлекс от фазы $\mathrm{Y}_{3} \mathrm{Fe}_{5} \mathrm{O}_{12}$ можно увидеть также на рентгенограмме при $x=0.6$ и $2 \Theta \sim 28^{\circ}$.

Представляет интерес проанализировать изменение параметров элементарной ячейки гексаферрита $\mathrm{BaFe}_{12-x} \mathrm{Y}_{x} \mathrm{O}_{19}$, представленных на рис. 4. На приведенных концентрационных зависимостях параметров элементарной ячейки $(a, c, v)$ видно скачкообразное их увеличение при $x=0.6$, которое, видимо, связано с деформационными процессами в структуре в связи с началом распада твердого раствора, а дальнейшее увеличение степени замещения $x$ приводит к умень-

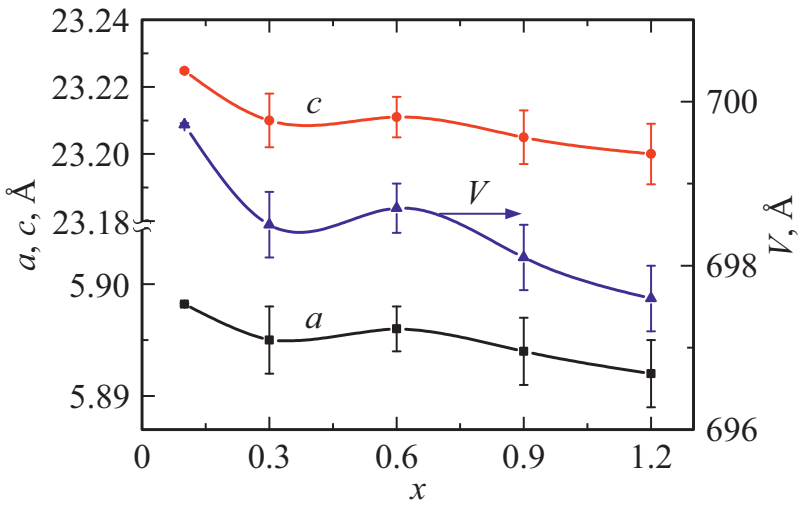

Рис. 4. Концентрационные зависимости параметров элементарной ячейки $\mathrm{BaFe}_{12-x} \mathrm{Y}_{x} \mathrm{O}_{19}(x=0.1-1.2)$.

шению всех параметров. Скачкообразное возрастание параметров при $x=0.6$ подтверждает предположение, сделанное выше, что пределом изоморфного вхождения ионов $\mathrm{Y}^{3+}$ является $x=0.6$.

C помощью магнитных измерений были получены зависимости удельной и остаточной намагниченностей, а также коэрцитивной силы от степени замещения $x$. На рис. 5, a приведены зависимости от $x$ удельной и остаточной намагниченностей. Они монотонны в интервале значений $x$ от 0.1 до 1.2. Оценивая изменение удельной намагниченности можно отметить, что в данном диапазоне она изменяется незначительно, всего на $9 \mathrm{emu} / \mathrm{g}$, что существенно меньше, чем для других замещающих элементов. Так, при замещении ионов $\mathrm{Fe}^{3+}$ ионами $\mathrm{Al}^{3+}[17]$ и $\mathrm{In}^{3+}[5]$ в том же диапазоне $\sigma_{s}$ уменьшилась на $18 \mathrm{emu} / \mathrm{g}$, а при вхождении $\mathrm{Ga}^{3+}$ - на $27 \mathrm{emu} / \mathrm{g}$ [17]. При этом коэрцитивная сила при замещении $\mathrm{Fe}^{3+}$ этими же элементами ведет себя несколько иначе, чем в случае иттрия (рис. $5, b$ ): при вхождении ионов $\mathrm{Y}^{3+}$ наблюдается рост $H_{c}$ с 2.4 до $3.14 \mathrm{kOe}$, а затем кривая выполаживается в диапазоне $x=0.9-1.2$, что согласуется с увеличением примесных фаз и уменьшением изоморфного иттрия в решетке гексаферрита. Если сопоставить изменение коэрцитивной силы при вхождении в решетку $\mathrm{Y}^{3+}$ со случаями $\mathrm{Al}^{3+}$ и $\mathrm{Ga}^{3+}$, то можно отметить следующее. Коэрцитивная сила при замещении ионами $\mathrm{Al}^{3+}$ увеличивается от 3.8 до $6.9 \mathrm{kOe}$, после чего уменьшается до $4.6 \mathrm{kOe}$, а при замещении ионами $\mathrm{Ga}^{3+} H_{c}$ уменьшается от 2 до $0.5 \mathrm{kOe}$. Анализируя поведение $H_{c}$, гексаферрит $\mathrm{BaFe}_{12-x} \mathrm{Al}_{x} \mathrm{O}_{19}$ можно отнести к магнитотвердым, а $\mathrm{BaFe}_{12-x} \mathrm{Ga}_{x} \mathrm{O}_{19}-$ к магнитомягким ферритам [17]. При таком поведении коэрцитивной силы гексаферрит $\mathrm{BaFe}_{12-x} \mathrm{Y}_{x} \mathrm{O}_{19}$ занимает промежуточное положение, ближе к магнитотвердым материалам. Угол $\Theta$ между магнитным моментом образцов и направлением вектора $\gamma$-излучения стабилен и составляет $56.1^{\circ}$, что указывает на изотропный характер образцов $\mathrm{BaFe}_{12-x} \mathrm{Y}_{x} \mathrm{O}_{19}$.

Сравнивая влияние на свойства гексаферрита ионов $\mathrm{Y}^{3+}, \mathrm{Al}^{3+}$ и $\mathrm{Ga}^{3+}$, следует учесть, что ионные радиусы 

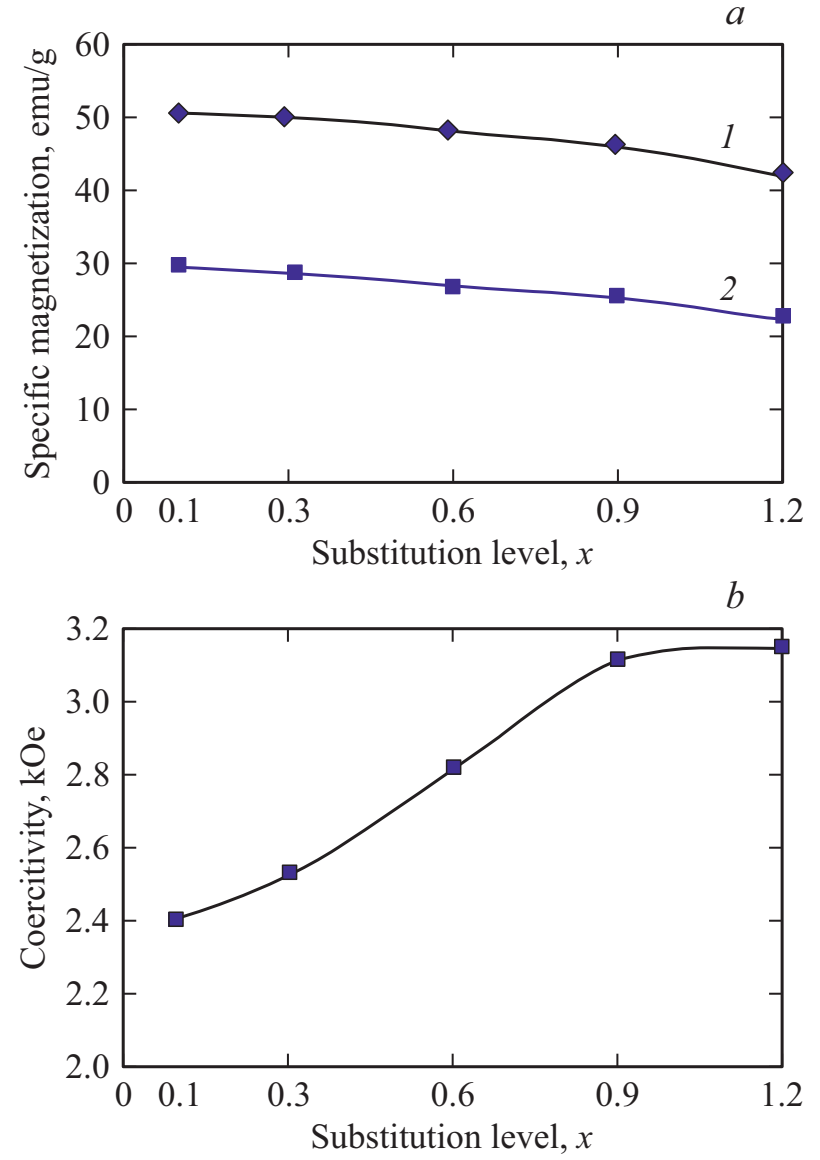

Рис. 5. Магнитные характеристики $\mathrm{BaFe}_{12-x} \mathrm{Y}_{x} \mathrm{O}_{19}: a-$ удельная намагниченность $\sigma_{s}(1)$ и остаточная намагниченность $\sigma_{r}(2) ; b-$ коэрцитивная сила.

как $\mathrm{Al}^{3+}$, так и $\mathrm{Ga}^{3+}$ значительно меньше ионного радиуса $\mathrm{Y}^{3+}$. Поэтому для сравнения, в первую очередь, магнитных свойств интересно сопоставить влияние изоморфных примесей с одинаковыми ионными радиусами. Таким ионом, сопоставимым с $\mathrm{Y}^{3+}$, является $\mathrm{In}^{3+}$. Для сравнения мы использовали некоторые результаты работы [5], где исследуются свойства гексаферрита $\mathrm{BaFe}_{12-x} \operatorname{In}_{x} \mathrm{O}_{19}$ с теми же содержаниями примесей и применены те же методы исследования.

Из сопоставления результатов исследования гексаферритов $\mathrm{BaFe}_{12-x} \mathrm{Y}_{x} \mathrm{O}_{19}$ и $\mathrm{BaFe}_{12-x} \mathrm{In}_{x} \mathrm{O}_{19}$ следует, что несмотря на одинаковые радиусы ионов $\mathrm{Y}^{3+}$ и $\mathrm{In}^{3+}$, магнитные свойства и структурные особенности гексаферритов существенно различаются. Изоморфная емкость гексаферрита $\mathrm{BaFe}_{12-x} \mathrm{In}_{x} \mathrm{O}_{19}$ существенно выше, нежели у $\mathrm{BaFe}_{12-x} \mathrm{Y}_{x} \mathrm{O}_{19}$. Катионные распределения также различаются. Если ионы $\mathrm{Y}^{3+}$ локализуются, в основном, в позиции $12 k$, то для ионов $\operatorname{In}^{3+}$ характерно неупорядоченное распределение в подрешетках $2 b, 4 f_{2}$ и $12 k$, что приводит к ослаблению межподрешеточных взаимодействий и неколлинеарной магнитной структуре. При этом нужно учитывать, что электронная конфигурация иона $\mathrm{Y}^{3+}-3 d^{10} 4 p^{6}$, a $\mathrm{In}^{3+}-4 d^{8} 5 s^{2}$, и это также является причиной различий магнитных свойств гексаферритов. Поэтому можно считать, что основной причиной разных свойств гексаферритов $\mathrm{BaFe}_{12-x} \mathrm{Y}_{x} \mathrm{O}_{19}$ и $\mathrm{BaFe}_{12-x} \operatorname{In}_{x} \mathrm{O}_{19}$ являются различное катионное распределение и разная электронная конфигурация ионов $\mathrm{Y}^{3+}$ и $\mathrm{In}^{3+}$.

Таким образом, проведенные с помощью мёссбауэровской спектроскопии, магнитометрии и рентгенографии исследования гексаферрита $\mathrm{BaFe}_{12-x} \mathrm{Y}_{x} \mathrm{O}_{19}$ показали, что изоморфная емкость гексаферрита $\mathrm{BaFe}_{12-x} \mathrm{Y}_{x} \mathrm{O}_{19}$ невысока, и начиная с $x=0.6$ в нем начинает происходить фазовое расслоение с образованием фаз $\mathrm{Y}_{3} \mathrm{Fe}_{5} \mathrm{O}_{12}$ и $\mathrm{BaFe}_{2} \mathrm{O}_{4}$. Данные мёссбауэровской спектроскопии показывают, что ионы $\mathrm{Y}^{3+}$ в изученном диапазоне замещений входят в позицию $12 k$ с образованием неэквивалентного положения $12 k^{\prime}$ за счет обрыва магнитных связей в триаде октаэдров $12 k \mathrm{Fe}(12 k)-\mathrm{O}-\mathrm{Fe}(12 k)$ и образования $\mathrm{Fe}(12 k)-\mathrm{O}-\mathrm{Y}(12 k)$. Сопоставление гексаферрита $\mathrm{BaFe}_{12-x} \mathrm{Y}_{x} \mathrm{O}_{19}$ с $\mathrm{BaFe}_{12-x} \mathrm{Al}_{x} \mathrm{O}_{19}$ и $\mathrm{BaFe}_{12-x} \mathrm{Ga}_{x} \mathrm{O}_{19}$, относящимися к магнитотвердым и магнитомягким разновидностям ферритов, показало, что иттрий-замещенный гексаферрит можно отнести к менее магнитотвердому, нежели алюмозамещенный. Сопоставление результатов исследования гексаферритов $\mathrm{BaFe}_{12-x} \mathrm{Y}_{x} \mathrm{O}_{19}$ и $\mathrm{BaFe}_{12-x} \operatorname{In}_{x} \mathrm{O}_{19}$ показало, что несмотря на одинаковые радиусы ионов $\mathrm{Y}^{3+}$ и $\operatorname{In}^{3+}$ их магнитные свойства существенно различаются, и основной причиной этого являются катионное распределение и разная электронная конфигурация ионов $\mathrm{Y}^{3+}$ и $\mathrm{In}^{3+}$.

\section{Финансирование работы}

Работа выполнена при поддержке Российского научного фонда (соглашение № 19-19-00694 от 06.05.2019).

\section{Конфликт интересов}

Авторы заявляют, что у них нет конфликта интересов.

\section{Список литературы}

[1] R.C. Pullar. Prog. Mater. Sci. 57, 1191 (2012).

[2] Ш.Ш. Башкиров, А.Б. Либерман, В.И. Синявский. Магнитная микроструктура ферритов. Изд-во Казан. ун-та, Казань (1978). $181 \mathrm{c}$.

[3] В.В. Коровушкин, А.В. Труханов, В.Г. Костишин, И.М. Исаев, И.В. Щетинин, Н.М. Дуров, А.Ю. Миронович, И.О. Минкова, К.А. Астапович. ФТТ 62, 5, 789 (2020).

[4] В.В. Коровушкин, М.Н. Шипко, В.Г. Костишин, И.М. Исаев, А.Ю. Миронович, С.В. Труханов, А.В. Труханов. Неорган. материалы 55, 10, 1065 (2019).

[5] В.В. Коровушкин, А.В. Труханов, М.Н. Шипко, В.Г. Костишин, И.М. Исаев, А.Ю. Миронович, С.В. Труханов. Журн. неорган. химии 64, 5, 463 (2019).

[6] А.В. Труханов, С.В. Труханов, В.Г. Костишин, Л.В. Панина, М.М. Салем, И.С. Казакевич, В.А. Турченко, В.В. Кочервинский, Д.А. Кривченя. ФТТ 59, 4, 721 (2017). 
[7] A.V. Trukhanov, M.A. Darwish, L.V. Panina, A.T. Morchenko, V.G. Kostishyn, V.A. Turchenko, D.A. Vinnik, E.L. Trukhanova, K.A. Astapovich, A.L. Kozlovskiy, M. Zdorovets, S.V. Trukhanov. J. Alloys Comp. 791, 522 (2019).

[8] M.A. Almessiere, Y. Slimani, H. Güngüne, A. Baykal, S.V. Trukhanov, A.V. Trukhanov. Nanomaterias 9, 1, 24 (2019).

[9] Y. Bakış, I.A. Auwal, B. Ünal, A. Baykal. Compos. B 99, 248 (2016).

[10] S. Güner, I.A. Auwal, A. Baykal, H. Sözeri. J. Magn. Magn. Mater. 416, 261 (2016).

[11] A. Auwal, H. Güngüneş, S. Güner, E. Sagar Shirsath, M. Sertkol, A. Baykal. Mater. Res. Bull. 80, 263 (2016).

[12] R. Topkaya, I. Auwal, A. Baykal. Ceram. Int. 42, 16296 (2016).

[13] K.M.Ur. Rehman, X. Liu, M. Li, S. Jiang, Y. Wu, C. Zhang, C. Liu, X. Meng, H. Li. J. Magn. Magn. Mater. 426, 183 (2017).

[14] R. Alam, M. Tehrani, M. Moradi, E. Hosseinpour, A. Sharbati. J.Magn. Magn. Mater. 323, 1040 (2011).

[15] F. Menil. J. Phys. Chem. Solids. 46, 7, 763 (1985).

[16] G.M. Bancroft, A.G. Maddok, R.G. Burns. Geochim. Cosmochim. Acta 31, 2219 (1967).

[17] V.G. Kostishyn, V.V. Korovushkin, I.M. Isaev, A.V. Trukhanov. East.-Eur. J. Enterp. Technol. 1, 10 (2017).

Редактор Е.Ю. Флегонтова 